\title{
Restricted posture in dentistry - a kinematic analysis of orthodontists
}

\author{
Daniela Ohlendorf ${ }^{*}$, Christina Erbe ${ }^{2}$, Imke Hauck¹, Jennifer Nowak¹, Ingo Hermanns ${ }^{3}$, Dirk Ditchen ${ }^{3}$, \\ Rolf Ellegast ${ }^{3}$ and David A. Groneberg ${ }^{1}$
}

\begin{abstract}
Background: This study aims at identifying orthodontic activities with the highest frequency of unfavorable/ awkward and static postures held over a period of more than $4 \mathrm{~s}$ based on kinematic analysis. Moreover, a separate analysis of static postures for orthodontic and non-orthodontic activities serves to evaluate the duration for which these particular postures are assumed.
\end{abstract}

Methods: In total, 21 (13f/8 m) orthodontists (age: $31.5 \pm 3.8$ years) participated in this study. CUELA, a personal measurement system, was used to collect kinematic data for all orthodontic activities in a working day. Angle values of the head and torso were evaluated in accordance with ergonomic standards. Only those postures that were held statically for $4 \mathrm{~s}$ and longer were selected for further analysis. Alongside the kinematic analysis, the activities performed on-site were also subject to a detailed computerized analysis. The synchronization of data collected from both measurements arranges the patterns of posture found chronologically and in conjunction with the orthodontic activities performed ((I) "treatment" (II) "office" and (III) "other activities").

Results: For (I) we observed an anterior inclination of the head and torso area as well as a twist of the head and neck area to the right. We found anterior back inclination and lateral back torsion to the right for (II) and (III). If, furthermore, we differentiate the duration of static postures, there are primarily short to medium-term (4-30s) static postures identified for (I). Also, categories (II) and (III) predominantly demonstrate static back postures with a duration of up to $30 \mathrm{~s}$. With regard to (II) we observed that the back is ventrally inclined for $10.1 \%$ of the total activity duration.

Conclusions: During treatment static strains are observed in the entire head and torso area. On the contrary, static postures prevalent in the torso area are essential for activities of the other categories, particularly office work. These findings allow for a careful selection of unfavorable and static postures for each of the activities performed and help to develop specific preventive measures.

Keywords: Restricted posture, Orthodontist, Cuela, Kinematic analysis

\section{Background}

Studies around the globe point to the high prevalence of musculoskeletal disorders in orthodontists regardless of their given work experience [1-8]. These studies show an increased pain symptomatology especially in the neck, shoulder, and/or back area caused by dental activities [9-15]. Moreover, Blanc et al. [16] found out that at different dental treatment units muscle activities and

\footnotetext{
* Correspondence: ohlendorf@med.uni-frankfurt.de

${ }^{1}$ Institute of Occupational Medicine, Social Medicine and Environmental Medicine, Goethe-University, Theodor-Stern-Kai 7, 60590 Frankfurt am Main, Germany

Full list of author information is available at the end of the article
}

the joint angles assumed vary with the type of occupational posture. As a result, there is evidence that musculoskeletal disorders and the pain caused often constrain the work of dentists and orthodontists $[1,17,2]$ or even force them to retire early on grounds of occupational disability $[18,19]$. Muscular dysbalances and the resulting disorders develop primarily due to poor occupational posture $[12,20,21]$. These disorders most likely originate in working continuously in static postures but also in often-repeated workflows [22, 17, 23]. Hereby, static refers to maintaining an unfavorable (restricted) posture which requires more static muscle activity 
and possibly results in excessive muscular strain as a consequence [24].

Various ergonomic concepts were developed to decrease musculoskeletal disorders. As early as 1972, Schön [25] discovered that static muscular fatigue occurs during dental activities but also stated that contrary to standing muscular fatigue decreases in a sitting position by $40 \%$ despite the increased pressure on the intervertebral discs. With ventral inflexion and rotation of the torso, a posture often assumed by dentists, the pressure on the spinal disc even increases by $400 \%$ [25]. Disorders caused by spinal disc issues can result in occupational disability [26-28].

Rohmert et al. [15] conclude that a change in posture to relax muscles is key to prevent signs of fatigue in the various muscle groups. An ideal approach to inhibit fatigue in the muscle groups is an exposition of $30 \mathrm{~s}$ followed by a micropause of a few seconds. In occupational medicine static posture is defined as any posture held for more than $4 \mathrm{~s}[29,30]$.

According to the surveys by Valachi et al. [26, 28] continuous static postures that involve more than $50 \%$ of the body muscles for stabilization present one of the main causes for musculoskeletal disorders in dentists and are thus considered more harmful to the human body than dynamic activities [26]. Previous studies used RULA (rapid upper limb assessment), for instance, to measure static postures in dentists [31, 32]. RULA serves to evaluate risk factors for job-related musculoskeletal disorders in the upper limbs. This method thereby measures static postures for a duration of more than one minute [31]. Park et al. [32] have demonstrated with RULA that the risk for dentists of developing musculoskeletal disorders is the highest in the low back and neck. Moreover, they found that the posture of dentists routinely comprises neck rotation, shoulder abduction, as well as a strong inclination of the torso to front [33].

Although, the routine of orthodontists differs greatly from the routine of dentists, only a few studies have been conducted to date to measure occupational posture in orthodontists [34]. Therefore, our objective is to study the orthodontic workday through kinematic analysis following the RULA method. The continued measurement of postures and joint angles allows for a concise quantification of occupational postures as restricted postures. This analysis also aims to reveal that the overall percentage of non-neutral postures is higher in the torso than in the head and neck area for all three categories ((I) "treatment", (II) "office" and (III) "other activities"). Moreover, the duration of static postures involved in the most common activities is also determined. Of particular interest here is whether the percentage of static postures held for more than $4 \mathrm{~s}$ is higher for treatment activities as opposed to office work by default.

\section{Methods}

\section{Subjects}

Twenty one $(13 \mathrm{f} / 8 \mathrm{~m})$ orthodontic postgraduate residents employed at dental schools in Germany participated in this study. The average age of subjects was $31.5 \pm 3.8$ years and their work experience accounted for $3.9 \pm 2.5$ years. One dropout was recorded for the group of male participants. Among others, exclusion criteria for participants were signs of functional impairments of the musculoskeletal system due to spinal fusion or severe deformities of the spine (e.g.: scoliosis). Furthermore, injuries of the musculoskeletal system such as disc herniation and fractures in the back and neck, as well as muscular diseases that occurred more than 2 years prior to the study were criteria for exclusion.

Each participant was measured on a randomly selected workday. This study was approved by the Ethics Committee (135/14) of Goethe University in Frankfurt am Main. All participants signed an informed consent to take part in the study.

\section{CUELA measuring system}

The CUELA system (computer-assisted acquisition and long-term analysis of stresses on the musculoskeletal system) was used to record the subjects' body postures $[35,36]$. CUELA is a personal system developed at the Institute for Occupational Safety and Health of the German Social Accident Insurance (IFA; Sankt Augustin/ Germany) that uses sensors (accelerometers [ADXL 103/ 203] and gyroscopes [muRata ENC-03R] for head, arms, legs, back, potentiometers [Contelect] for back torsion) to measure the position or angle, and, in this way, allows for a kinematic reconstruction of the subjects' motions. CUELA detected the probable degrees of freedom essential for a realistic description of dynamic motions at a sampling frequency of $50 \mathrm{~Hz}$ and with an angular accuracy of $\pm 1^{\circ}$. Please refer to Table 1 for all calculated angle values [37-39].

\section{Measuring system: Objective work activity analysis with mini-PC}

Observers use software specifically developed for work activity analysis to document the workflows of orthodontists in real time by the second on a portable hand-held computer (UMPC Samsung Q1, Samsung Electronics GmbH. Schwalbach, Germany). On the one hand, this approach will allow for identifying the particular work activity, whereas, on the other hand, the software can also determine the duration of these activities within these workflows. For a more detailed description of the system please refer the methods paper by Mache et al. [40, 41]. 
Table 1 Depiction of the recorded body/joint angles based on DIN-EN 1005-4, applied evaluation parameters and assessment criteria according to ergonomic layouts

\begin{tabular}{|c|c|c|c|}
\hline Body areas & Joint/Body area & $\begin{array}{l}\text { Degree of freedom according to medical } \\
\text { Definitions (posture concept) }\end{array}$ & $\begin{array}{l}\text { Angle range according to ergonomic } \\
\text { standards }\end{array}$ \\
\hline \multirow[t]{4}{*}{ Head/neck } & \multirow[t]{2}{*}{ Head } & flexion/extension $\left(\mathrm{H} \_\mathrm{f}\right)(44)$ & $\begin{array}{l}\text { Neutral: } 0 \text { to } 25^{\circ} \\
\text { Moderate: } 25 \text { to } 85^{\circ} \\
\text { Awkward: }<0^{\circ} \&>85^{\circ}\end{array}$ \\
\hline & & lateral inclination (H_li) (44) & $\begin{array}{l}\text { Neutral: }-10 \text { to } 10^{\circ} \\
\text { Awkward: }<-10^{\circ} \&>10^{\circ}\end{array}$ \\
\hline & \multirow[t]{2}{*}{ Cervical spine (CS) } & flexion/extension (CS_f) (44) & $\begin{array}{l}\text { Neutral: } 0 \text { to } 25^{\circ} \\
\text { Awkward: }<0^{\circ} \&>25^{\circ}\end{array}$ \\
\hline & & lateral flexion (CS_If) (44) & $\begin{array}{l}\text { Neutral: }-10 \text { to } 10^{\circ} \\
\text { Awkward: }<-10^{\circ} \&>10^{\circ}\end{array}$ \\
\hline \multirow[t]{7}{*}{ Back } & \multirow[t]{2}{*}{ Thoracic spine (TS) } & flexion/extension (TS_f) (44) & $\begin{array}{l}\text { Neutral: } 0 \text { to } 20^{\circ} \\
\text { Moderate: } 20 \text { to } 60^{\circ} \\
\text { Awkward: }<0^{\circ} \&>60^{\circ}\end{array}$ \\
\hline & & lateral flexion (TS_If) (44) & $\begin{array}{l}\text { Neutral: }-10 \text { to } 10^{\circ} \\
\text { Moderate: }-10 \text { to }-20^{\circ} \\
\text { Moderate: } 10 \text { to } 20^{\circ} \\
\text { Awkward: }<-20^{\circ} \&>20\end{array}$ \\
\hline & \multirow[t]{2}{*}{ Lumbar spine (LS) } & flexion/extension (LS_f) & \multirow[t]{2}{*}{ No ergonomic layout available } \\
\hline & & lateral flexion (LS_If) & \\
\hline & \multirow[t]{3}{*}{ Torso (T) } & flexion/extension (T_f) (44) & $\begin{array}{l}\text { Neutral: } 0 \text { to } 20^{\circ} \\
\text { Moderate: } 20 \text { to } 40^{\circ} \\
\text { Awkward: }<0^{\circ} \&>40^{\circ}\end{array}$ \\
\hline & & Inclination (T_i) (44) & $\begin{array}{l}\text { Neutral: } 0 \text { to } 20^{\circ} \\
\text { Moderate: } 20 \text { to } 60^{\circ} \\
\text { Awkward: }<0^{\circ} \&>60^{\circ}\end{array}$ \\
\hline & & $\begin{array}{l}\text { Lateral inclination (T_li) (44) } \\
\text { torsion (T_t) (44) }\end{array}$ & $\begin{array}{l}\text { Neutral: }-10 \text { to } 10^{\circ} \\
\text { Moderate: }-10 \text { to }-20^{\circ} \\
\text { Moderate: } 10 \text { to } 20^{\circ} \\
\text { Awkward: }<-20^{\circ} \&>20^{\circ}\end{array}$ \\
\hline
\end{tabular}

\section{Experimental design}

Prior to the study, the software was programmed in accordance with the work activity spectrum of orthodontists. All activities were divided into the categories (I) "treatment," (II) "office," and (III) "other activities." These categories serve to describe all activities involved in the day-to-day routine of orthodontists (Table 2). The following figures illustrate examples of assigned activities of categories I and III (Fig. 1).

\section{Evaluation}

Synchronizing the work activity analysis with the CUELA measurement in the CUELA software (IFA; Sankt Augustin/Germany) enables a temporal allocation of the motion patterns found and the associated activities. Relevance and duration of each work category were divided according to their percentage values. Angle values for each anatomical area (evaluation parameter) were then evaluated in compliance with ergonomic standards and assigned to a color-coded angle range representing ergonomic standards (traffic light: system red/ yellow/green). Based on the respective colors postures are assessed as unfavorable, moderate (acceptable with reservations), or neutral [30, 42, 43] (Table 1). In reference with the criteria for classification the percentage for each evaluation parameter is calculated and assessed with regard to whether activities are executed in neutral, moderate, or unfavorable postures for all activities (categories I, II, and III). Then, the percentage of moderate and unfavorable postures are added up and presented in summary as values that represent non-neutral postures.

The overall statistics show the percentage of static non-neutral postures for the respective activities and for each evaluation parameter. Static postures are those postures that are ranked as moderate or unfavorable according to ergonomic standards as outlined by ISO standards [30] and held for more than $4 \mathrm{~s}$. As postures can be held for a longer duration, the RULA method (rapid upper limb assessment) [31] has been used to apply a posture related screening method for static postures among dentists for durations of more than $1 \mathrm{~min}$ without further differentiation.

Based on these valuation methods for static postures we also distinguish in addition to the $\geq 4 \mathrm{~s}$ threshold between postures that are held for more than $60 \mathrm{~s}$, between 30 and $60 \mathrm{~s}$, and postures that are held between 4 
Table 2 Depiction of all categories with the respective work stages, their definition and the respective duration

\begin{tabular}{|c|c|c|}
\hline Category & Sub-activities & Definition \\
\hline \multirow[t]{15}{*}{ Treatment } & impressions & Taking an impression of the patient (teeth) \\
\hline & consultation & Case discussion of two doctors on patients \\
\hline & Mini Implant & Insertion of a mini implant \\
\hline & archwire/elastic change & Replacement of archwire/elastics \\
\hline & photo & Case documentation with the camera \\
\hline & craft activities & Generic term for operations that do not fall into the above activities \\
\hline & removable appliance & Insertion/control of removable appliances \\
\hline & conservative dentistry & Cosmetic restorative filling (dental work) \\
\hline & fixed appliance & Bonding/separation/repair of fixed appliances (mostly multibracket appliance) \\
\hline & palpation & Palpation of the muscles of the face/temporomandibular joints of the patient \\
\hline & break & Short breaks during treatment \\
\hline & prophylaxis & Cleaning of teeth and brushing training \\
\hline & splint & Insertion/control of occlusal appliance (splint) \\
\hline & medical examination & First time/control examination of a patient \\
\hline & angle piece/ultrasound & Usage of angle piece/ultrasound handpiece during the treatment \\
\hline \multirow[t]{4}{*}{ Office } & file & Inspection of records (Findings/Dental Model/X-ray image) \\
\hline & Office work & Data entry into patient record/PC Work \\
\hline & model analysis & Analysis and design of the treatment plan using dental casts/X-ray images \\
\hline & phone call & Phone conversations \\
\hline \multirow[t]{6}{*}{ Other activities } & meeting & Medical meetings \\
\hline & conversation & Discussions with patients and staff as solitary activities \\
\hline & hygiene & Hygiene (washing/disinfecting hands, putting on gloves/mouth protection) \\
\hline & Taking up/putting down of instruments & Take instruments from a drawer/store within and also before/after treatment \\
\hline & laboratory & Any laboratory work \\
\hline & walk & Covering distances \\
\hline
\end{tabular}

and $10 \mathrm{~s}$ (statics components) [29, 30]. Furthermore, we calculate the quotient based on the total percentage of static and non-neutral postures to determine the percentage of static postures involved in non-neutral postures (total percentage of static non-neutral postures).

\section{Results}

We were able to use a total of $95.9 \mathrm{~h}(5752.6 \mathrm{~min})$ of data material excluding neutral postures and non-related activities such as breaks or toileting. Category I account for 34\% (1952.9 $\mathrm{min}$ ), category II "office" for 33\% (1893.7 $\mathrm{min}$ ) and category III "other activities" for 33\%, (1906.3 $\mathrm{min}$ ) of the total data material.

With regard to treatment (I) the study focused on the most frequently executed activities such as "craft activities," "archwire/elastic change," "contra-angle/ ultrasound," "medical examination," "fixed appliance" and "removable appliance." Of these six sub-activities "archwire/elastic change" accounts for $705.3 \mathrm{~min}(36 \%)$, "craft activities" for $408 \mathrm{~min}$ (21\%), and "fixed appliance" for $325 \mathrm{~min}$ (17\%) $74 \%$ of the total treatment time.
The analysis of kinematic data mainly focuses on nonneutral motions with a percentage of $\geq 50 \%$ of the total activity duration and a conspicuous percentage of static postures of $\geq 25 \%$. If there were no anomalies found for the total percentage of static postures, data material for non-neutral postures of $\geq 75 \%$ was analyzed. These threshold areas were determined in relation to priority rankings.

Tables 3 and 5 show the percentage of postures for activities of the categories (I), (II), and (III) divided into ergonomic classifications (neutral, moderate, unfavorable). Moreover, these tables list the sum values derived from adding results for moderate and unfavorable postures as non-neutral postures. Tables 4 and 6 refer to the total percentage of static postures, the temporal differentiation, as well as the total percentage of static nonneutral postures.

\section{Category I: Treatment}

In the head and neck area the percentage of non-neutral postures with head flexio/extension $\left(\mathrm{H}_{-} \mathrm{f}\right)$ during the 

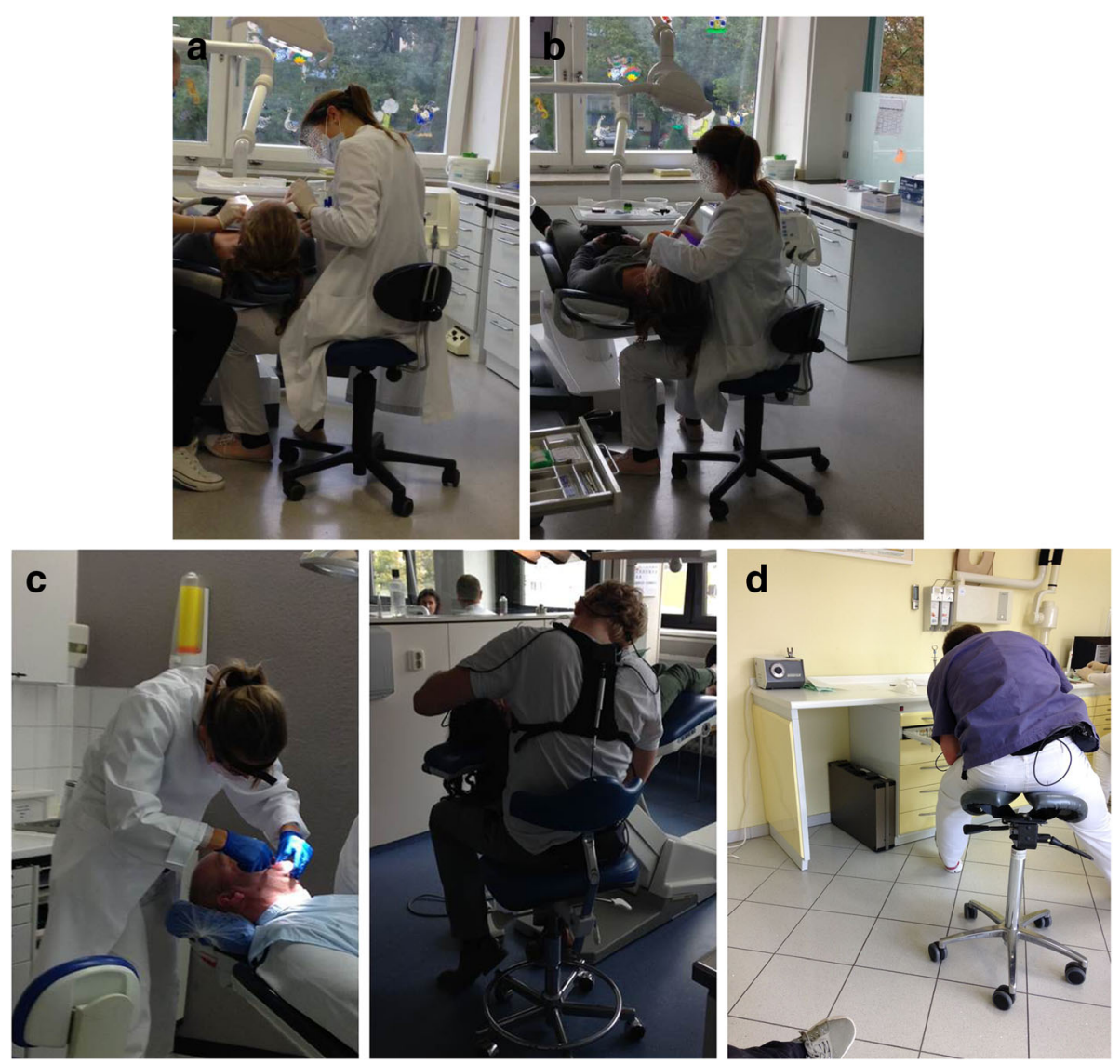

Fig. 1 a Category I: Carry out craft activities. b Category I: Bonding of fixed appliances. c: Category I: Medical examination on a patient. d: Category III: Taking instruments

activities "archwire/elastic change" and "craft activities" is at $82.6 \%$ or $76.4 \%$, from which $15.4 \%$ or $18.5 \%$ are performed statically. The percentage of static postures for both activities is between 4 and $10 \mathrm{~s}$ at $12.6 \%$ and $11.1 \%$ and thus accounts for the largest share in this category. $90.4 \%$ of postures in the non-neutral range observed during the activity "contra-angle/ultrasound" demonstrated a percentage of static postures of $46.9 \%$. Regarding static postures (42.9\%) $21.1 \%$ are held between 4 and $10 \mathrm{~s}$ and $21 \%$ are held for $10-30 \mathrm{~s}$.

During the activity "contra-angle/ultrasound" lateral inclination of the head $\left(\mathrm{H}_{-} \mathrm{li}\right)$ demonstrates a percentage of non-neutral postures at $68.1 \%$ with an overall percentage of static postures of $40.5 \%$. Hereby, postures that last between 4 and $10 \mathrm{~s}$ account for $16.9 \%$, and thus the largest percentage of static postures, followed by $11.5 \%$ of postures that last for 10-30 s.

For the same activity we observed conspicuous values with regard to the extension/flexion of the cervical spine (CS_f) and the lateral flexion (H_li). With cervical flexion/ extension (CS_f) the percentage of non-neutral postures is at $59.6 \%$ with a total percentage of static postures of $47.4 \%$.
With regard to the total percentage of static postures of $34.8 \%$ we find that $13.1 \%$ of postures are held between 4 and $10 \mathrm{~s}, 19.2 \%$ between 10 and $30 \mathrm{~s}$, and $2.5 \%$ last between 30 and $60 \mathrm{~s}$. The percentage of non-neutral postures for cervical spine lateral flexion (CS_lf) is $61.8 \%$ and accounts for $38.7 \%$ of the total percentage of static postures. The duration of static postures lies between 4 and $10 \mathrm{~s}(13.9 \%)$ and between 10 and $30 \mathrm{~s}(11.4 \%)$.

In the back area we found conspicuous results for flexion/extension of the thoracic spine (TS_f) regarding the sub-activities "archwire/elastic change," "medical examination," and "fixed appliance." During "archwire/ elastic change" and "fixed appliance" the percentage of non-neutral postures is $50.7 \%$ or $55 \%$, which accounts for $49.7 \%$ or $53.3 \%$ of the overall percentage of nonneutral postures. $15.3 \%$ or $15.6 \%$ of static postures last between 4 and $10 \mathrm{~s}$ while $12.3 \%$ or $12.1 \%$ have a duration of $10-30 \mathrm{~s}$. The percentage of non-neutral postures for "medical examination" is $54.8 \%$ and comprises $34.6 \%$ of the total percentage of static postures. Considering the overall allocation of static postures $(24.5 \%)$, the percentage of static postures that are held between 4 and 
Table 3 Treatment: Percentage of neutral, moderate, and unfavorable/awkward postures (\%) of the total activity duration for the head and neck area as well as the percentage of non-neutral postures as the sum of all moderate and unfavorable/awkward postures (\%). See Table 1 for color-coded ranking system

\begin{tabular}{|c|c|c|c|c|c|c|}
\hline Posture concept $\left[^{\circ}\right]$ & Activity & $\begin{array}{r}\text { Duration } \\
{[\text { [min] }}\end{array}$ & $\begin{array}{r}\begin{array}{r}\text { Percentage } \\
\text { of non- } \\
\text { neutral } \\
\text { postures [\% } \\
1\end{array} \\
\end{array}$ & $\begin{array}{r}\begin{array}{r}\text { Percentage of } \\
\text { neutral }\end{array} \\
\text { postures [\%] }\end{array}$ & $\begin{array}{r}\text { Percentage of } \\
\text { moderate } \\
\text { postures [\%] }\end{array}$ & $\begin{array}{r}\text { Percentage of } \\
\text { awkward } \\
\text { postures [\%] }\end{array}$ \\
\hline \multirow{6}{*}{$\begin{array}{l}\text { Head flexion } \\
\quad\left(\mathrm{H} \_\mathrm{f}\right)\end{array}$} & archwire / elastics change & 705.3 & 82.6 & 17.4 & 78.4 & 4.2 \\
\hline & craft activities & 408.25 & 72.4 & 27.6 & 68.2 & 4.2 \\
\hline & removable appliance & 83.2 & 57.2 & 42.8 & 45.8 & 11.4 \\
\hline & fixed appliance & 325 & 76.4 & 23.6 & 72.4 & 4 \\
\hline & medical examination & 228.3 & 73.8 & 26.2 & 68.7 & 5.1 \\
\hline & contra-angle/ ultrasound & 202.8 & 90.4 & 9.6 & 83.8 & 6.6 \\
\hline \multirow{6}{*}{$\begin{array}{l}\text { Head lateral inclination } \\
\text { (H_li) }\end{array}$} & archwire / elastics change & 705.3 & 55.9 & 44.1 & & 55.9 \\
\hline & craft activities & 408.25 & 50.2 & 49.8 & & 50.2 \\
\hline & removable appliance & 83.2 & 54.7 & 45.3 & & 54.7 \\
\hline & fixed appliance & 325 & 57.1 & 42.9 & & 57.1 \\
\hline & medical examination & 228.3 & 59.7 & 40.3 & & 59.7 \\
\hline & contra-angle/ ultrasound & 202.8 & 68.1 & 31.9 & & 68.1 \\
\hline \multirow{6}{*}{$\begin{array}{l}\text { Cervical spine } \\
\text { flexion/extension } \\
\text { (CS_f) }\end{array}$} & archwire / elastics change & 705.3 & 57.2 & 42.8 & & 57.2 \\
\hline & craft activities & 408.25 & 47.2 & 52.8 & & 47.2 \\
\hline & removable appliance & 83.2 & 40 & 60 & & 40 \\
\hline & fixed appliance & 325 & 47.5 & 52.5 & & 47.5 \\
\hline & medical examination & 228.3 & 46.7 & 53.3 & & 46.7 \\
\hline & contra-angle/ ultrasound & 202.8 & 59.6 & 40.4 & & 59.6 \\
\hline \multirow{6}{*}{$\begin{array}{l}\text { Cervical spine lateral } \\
\text { flexion (CS_lf) }\end{array}$} & archwire / elastics change & 705.3 & 48.4 & 51.6 & & 48.4 \\
\hline & craft activities & 408.25 & 47.8 & 52.2 & & 47.8 \\
\hline & removable appliance & 83.2 & 43.6 & 56.4 & & 43.6 \\
\hline & fixed appliance & 325 & 52.2 & 47.8 & & 52.2 \\
\hline & medical examination & 228.3 & 51.2 & 48.8 & & 51.2 \\
\hline & contra-angle/ ultrasound & 202.8 & 61.8 & 38.2 & & 61.8 \\
\hline \multirow{6}{*}{$\begin{array}{c}\text { Thoracic spine } \\
\text { flexion/extension } \\
\text { (TS_f) }\end{array}$} & archwire / elastics change & 705.3 & 50.7 & 49.3 & 50.5 & 0.2 \\
\hline & craft activities & 408.25 & 43.4 & 56.6 & 42.1 & 1.3 \\
\hline & removable appliance & 83.2 & 45.1 & 54.9 & 42.7 & 2.4 \\
\hline & fixed appliance & 325 & 55 & 45 & 54.7 & 0.2 \\
\hline & medical examination & 228.3 & 54.8 & 45.2 & 52.2 & 2.6 \\
\hline & contra-angle/ ultrasound & 202.8 & 65.5 & 34.5 & 65.3 & 0.2 \\
\hline \multirow{6}{*}{$\begin{array}{l}\text { Thoracic spine } \\
\text { Lateral flexion (TS_lf) }\end{array}$} & archwire / elastics change & 705.3 & 19.3 & 80.7 & 17.9 & 1.4 \\
\hline & craft activities & 408.25 & 13.3 & 86.7 & 12.3 & 1 \\
\hline & removable appliance & 83.2 & 12 & 88 & 11.2 & 0.8 \\
\hline & fixed appliance & 325 & 10.8 & 89.2 & 10.1 & 0.7 \\
\hline & medical examination & 228.3 & 22 & 78 & 18.6 & 3.5 \\
\hline & contra-angle/ ultrasound & 202.8 & 13.9 & 86.1 & 13.5 & 0.4 \\
\hline \multirow{6}{*}{$\begin{array}{c}\text { Torso } \\
\text { flexion/extension (T_f })\end{array}$} & archwire / elastics change & 705.3 & 73.5 & 26.5 & 54.5 & 19 \\
\hline & craft activities & 408.25 & 67.5 & 32.5 & 44.6 & 22.9 \\
\hline & removable appliance & 83.2 & 72.1 & 27.9 & 50.7 & 21.4 \\
\hline & fixed appliance & 325 & 71.5 & 28.5 & 43 & 28.4 \\
\hline & medical examination & 228.3 & 75.3 & 24.7 & 48.6 & 26.7 \\
\hline & contra-angle/ ultrasound & 202.8 & 69.9 & 30.1 & 37.2 & 32.7 \\
\hline \multirow{6}{*}{ Torso inclination $\left(\mathrm{T} \_\mathrm{i}\right.$ ) } & archwire / elastics change & 705.3 & 4.7 & 95.3 & 4.7 & \\
\hline & craft activities & 408.25 & 7.4 & 92.6 & 7.4 & \\
\hline & removable appliance & 83.2 & 13.6 & 86.4 & 13.5 & 0.2 \\
\hline & fixed appliance & 325 & 14.3 & 85.7 & 14.3 & \\
\hline & medical examination & 228.3 & 9.7 & 90.3 & 9.7 & 0.1 \\
\hline & contra-angle/ ultrasound & 202.8 & 16.1 & 83.9 & 16.1 & 0.1 \\
\hline \multirow{6}{*}{$\begin{array}{c}\text { Torso lateral } \\
\text { inclination (T_li) }\end{array}$} & archwire / elastics change & 705.3 & 13.4 & 86.6 & 13 & 0.4 \\
\hline & craft activities & 408.25 & 7.8 & 92.2 & 7.4 & 0.4 \\
\hline & removable appliance & 83.2 & 8 & 92 & 7.7 & 0.3 \\
\hline & fixed appliance & 325 & 7.1 & 92.9 & 6.8 & 0.3 \\
\hline & medical examination & 228.3 & 15.5 & 84.5 & 13.5 & 2 \\
\hline & contra-angle/ ultrasound & 202.8 & 6.2 & 93.8 & 6 & 0.1 \\
\hline \multirow{6}{*}{ Torso torsion (T_t $)$} & archwire / elastics change & 705.3 & 31.7 & 68.3 & 26.3 & 5.4 \\
\hline & craft activities & 408.25 & 26.7 & 73.3 & 20.4 & 6.3 \\
\hline & removable appliance & 83.2 & 36.1 & 63.9 & 22.7 & 13.5 \\
\hline & \begin{tabular}{|l|} 
fixed appliance \\
\end{tabular} & 325 & 27.9 & 72.1 & 22.4 & 5.4 \\
\hline & medical examination & 228.3 & 36.3 & 63.7 & 26.3 & 10 \\
\hline & contra-angle/ ultrasound & 202.8 & 33.8 & 66.2 & 23.7 & 10.1 \\
\hline
\end{tabular}

$10 \mathrm{~s}$ accounts with $16.1 \%$ for the largest share, followed by $8.2 \%$ of static postures that are held for $10-30 \mathrm{~s}$.

With torso flexion/extension ( $T_{-} f$ ) we found conspicuous values regarding the percentage of non-neutral postures for all activities performed $(67.5 \%-75.3 \%)$ as well as a conspicuous total percentage of static postures between $28.5 \%-56.5 \%$. $10.6 \%-21.7 \%$ of static postures have a duration of $4-10 \mathrm{~s}, 10.5 \%$ - 25\% last between 10 
Table 4 Percentage of static postures $\geq 4$ s during treatment. Figure legend: total percentage of static postures $=$ sum of all moderate and unfavorable/awkward postures that occur with all activities

\begin{tabular}{|c|c|c|c|c|c|c|c|c|c|}
\hline $\begin{array}{c}\text { Posture } \\
\text { concept } \\
{\left[{ }^{\circ}\right]}\end{array}$ & Activity & $\begin{array}{c}\text { Duratio } \\
\text { n } \\
\text { [min] }\end{array}$ & \begin{tabular}{|c|} 
Static \\
postures \\
$4-10$ s \\
{$[\%]$}
\end{tabular} & $\begin{array}{c}\text { Static } \\
\text { postures1 } \\
\mathbf{0 - 3 0 s}[\%]\end{array}$ & \begin{tabular}{|c|} 
Static \\
postures \\
30-60s \\
{$[\%]$}
\end{tabular} & \begin{tabular}{|c|} 
Static \\
posture \\
$\mathrm{s} \geq 6 \mathrm{~s}$ \\
{$[\%]$} \\
{$[\%]$}
\end{tabular} & \begin{tabular}{|c|c|} 
Total \\
percentag \\
e of static \\
postures \\
{$[\%]$} \\
\end{tabular} & 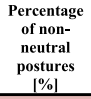 & $\begin{array}{c}\text { Total } \\
\text { percentage of } \\
\text { static non- } \\
\text { neutral } \\
\text { postures [\%] } \\
\end{array}$ \\
\hline \multirow{6}{*}{$\begin{array}{l}\text { Head flexion } \\
\text { (H_f) }\end{array}$} & $\begin{array}{l}\begin{array}{l}\text { archwire / elastics } \\
\text { change }\end{array} \\
\end{array}$ & 705.3 & 12.6 & 2.9 & & & 15.4 & 82.6 & 18.4 \\
\hline & \begin{tabular}{|l|} 
craft activities \\
\end{tabular} & 408.25 & 11.1 & 3.4 & 0.3 & & 14.8 & 72.4 & 18.7 \\
\hline & \begin{tabular}{|l|}
$\begin{array}{l}\text { removable } \\
\text { appliance }\end{array}$ \\
\end{tabular} & 83.2 & 3 & 0.3 & & & 3.2 & 57.2 & 6.4 \\
\hline & fixed appliance & 325 & 14.3 & 3.9 & 0.2 & & 18.5 & 76.4 & 23.6 \\
\hline & \begin{tabular}{|l|} 
medical \\
examination
\end{tabular} & 228.3 & 8.8 & 1.8 & & & 10.6 & 73.8 & 12.7 \\
\hline & $\begin{array}{l}\text { contra-angle/ } \\
\text { ultrasound }\end{array}$ & 202.8 & 21.1 & 21 & 0.8 & & 42.9 & 90.4 & 46.9 \\
\hline \multirow{6}{*}{$\begin{array}{c}\text { Head lateral } \\
\text { inclination } \\
\text { (H_li) }\end{array}$} & $\begin{array}{l}\begin{array}{l}\text { archwire / elastics } \\
\text { change }\end{array} \\
\end{array}$ & 705.3 & 6.6 & 1.2 & & & 7.8 & 55.9 & 13.5 \\
\hline & \begin{tabular}{|l|} 
craft activities \\
\end{tabular} & 408.25 & 6.8 & 2.3 & & & 9.2 & 50.2 & 15.5 \\
\hline & \begin{tabular}{|l|}
$\begin{array}{l}\text { removable } \\
\text { appliance }\end{array}$ \\
\end{tabular} & 83.2 & 3.8 & 1.6 & & & 5.4 & 54.7 & 9 \\
\hline & fixed appliance & 325 & 9.3 & 3.2 & 0.4 & & 12.9 & 57.1 & 22.3 \\
\hline & \begin{tabular}{|l|} 
medical \\
examination
\end{tabular} & 228.3 & 5.6 & 1.2 & & & 6.9 & 59.7 & 9.8 \\
\hline & \begin{tabular}{|l|} 
contra-angle// \\
ultrasound
\end{tabular} & 202.8 & 16.9 & 11.5 & 0.5 & & 29 & 68.1 & 40.5 \\
\hline \multirow{6}{*}{$\begin{array}{l}\text { Cervical spine } \\
\text { flexion/extensi } \\
\text { on (CS_f })\end{array}$} & $\begin{array}{l}\begin{array}{l}\text { archwire / lastics } \\
\text { change }\end{array} \\
\end{array}$ & 705.3 & 10.7 & 2.9 & 0.1 & & 13.6 & 57.2 & 22 \\
\hline & craft activities & 408.25 & 8.4 & 3.1 & 0.2 & & 11.6 & 47.2 & 20.5 \\
\hline & \begin{tabular}{|l|}
$\begin{array}{l}\text { removable } \\
\text { appliance }\end{array}$ \\
\end{tabular} & 83.2 & 1.8 & 0.2 & & & 2 & 40 & 4.1 \\
\hline & $\begin{array}{l}\text { fixed appliance } \\
\end{array}$ & 325 & 9.7 & 4 & 0.2 & & 13.8 & 47.5 & 25.3 \\
\hline & \begin{tabular}{|l|}
$\begin{array}{l}\text { medical } \\
\text { examination }\end{array}$ \\
\end{tabular} & 228.3 & 6.6 & 1.6 & & & 8.2 & 46.7 & 13.3 \\
\hline & \begin{tabular}{|l|}
$\begin{array}{l}\text { contra-angle/ } \\
\text { ultrasound }\end{array}$ \\
\end{tabular} & 202.8 & 13.1 & 19.2 & 2.5 & & 34.8 & 59.6 & 47.4 \\
\hline \multirow{6}{*}{$\begin{array}{l}\text { Cervical spine } \\
\text { lateral flexion } \\
\text { (CS_lf) }\end{array}$} & $\begin{array}{l}\begin{array}{l}\text { archwire / elastics } \\
\text { change }\end{array} \\
\end{array}$ & 705.3 & 5.9 & 1 & & & 6.9 & 48.4 & 13.4 \\
\hline & \begin{tabular}{|c|} 
craft activities \\
\end{tabular} & 408.25 & 7 & 2.1 & & & 9.1 & 47.8 & 16.3 \\
\hline & \begin{tabular}{|l|} 
removable \\
appliance
\end{tabular} & 83.2 & 2.9 & 1.5 & & & 4.4 & 43.6 & 10.3 \\
\hline & \begin{tabular}{|l|} 
fixed appliance \\
\end{tabular} & 325 & 8 & 2.4 & 0.2 & & 10.6 & 52.2 & 19.8 \\
\hline & \begin{tabular}{|l|}
$\begin{array}{l}\text { medical } \\
\text { examination }\end{array}$ \\
\end{tabular} & 228.3 & 5.3 & 1 & 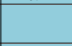 & & 6.3 & 51.2 & 9.2 \\
\hline & \begin{tabular}{|l|}
$\begin{array}{l}\text { contra-angle/ } \\
\text { ultrasound }\end{array}$ \\
\end{tabular} & 202.8 & 13.9 & 11.4 & 0.3 & & 25.5 & 61.8 & 38.7 \\
\hline \multirow{6}{*}{$\begin{array}{l}\text { Thoracic spine } \\
\text { flexion/extensi } \\
\text { on (TS_f) }\end{array}$} & \begin{tabular}{|l|}
$\begin{array}{l}\text { archwire / elastics } \\
\text { change }\end{array}$ \\
\end{tabular} & 705.3 & 15.3 & 12.3 & 0.9 & 0.4 & 28.9 & 50.7 & 49.7 \\
\hline & \begin{tabular}{|l|} 
craft activities \\
\end{tabular} & 408.25 & 12.5 & 9 & 1.1 & & 22.6 & 43.4 & 40.7 \\
\hline & \begin{tabular}{|l|} 
removable \\
appliance
\end{tabular} & 83.2 & 7.6 & 2.9 & & & 10.5 & 45.1 & 18.1 \\
\hline & \begin{tabular}{|l|l|l|} 
fixed appliance \\
\end{tabular} & 325 & 15.6 & 12.1 & 1 & 1.2 & 29.9 & 55 & 53.3 \\
\hline & \begin{tabular}{|l|}
$\begin{array}{l}\text { medical } \\
\text { examination }\end{array}$ \\
\end{tabular} & 228.3 & 16.1 & 8.2 & 0.2 & & 24.5 & 54.8 & 34.6 \\
\hline & \begin{tabular}{|l|}
$\begin{array}{l}\text { contra-angle/ } \\
\text { ultrasound }\end{array}$ \\
\end{tabular} & 202.8 & 11 & 26.7 & 10 & 1.6 & 49.3 & 65.5 & 66.4 \\
\hline \multirow{6}{*}{$\begin{array}{l}\text { Thoracic spine } \\
\text { Lateral flexion } \\
\text { (TS_lf) }\end{array}$} & $\begin{array}{l}\begin{array}{l}\text { archwire / elastics } \\
\text { change }\end{array} \\
\end{array}$ & 705.3 & 5.9 & 4.4 & 0.5 & 0.1 & 10.9 & 19.3 & 42.9 \\
\hline & craft activities & 408.25 & 3 & 2.9 & 0.6 & 0.3 & 6.9 & 13.3 & 22.6 \\
\hline & \begin{tabular}{|l|} 
removable \\
appliance
\end{tabular} & 83.2 & 3.3 & 1.1 & & & 4.4 & 12 & 14 \\
\hline & \begin{tabular}{|l|} 
fixed appliance \\
\end{tabular} & 325 & 2.6 & 2.7 & 0.2 & 0.3 & 5.8 & 10.8 & 35.8 \\
\hline & \begin{tabular}{|l|} 
medical \\
examination
\end{tabular} & 228.3 & 6.7 & 4.3 & & & 11 & 22 & 31 \\
\hline & \begin{tabular}{|l|} 
contra-angle/ \\
ultrasound
\end{tabular} & 202.8 & 1.3 & 3.3 & 2.7 & 1.8 & 9.1 & 13.9 & 31.2 \\
\hline \multirow{6}{*}{$\begin{array}{c}\text { Torso } \\
\text { flexion/extensi } \\
\text { on (T_f })\end{array}$} & $\begin{array}{l}\begin{array}{l}\text { archwire / elastics } \\
\text { change }\end{array} \\
\end{array}$ & 705.3 & 20.7 & 17.8 & 1.9 & 0.5 & 41 & 73.5 & 54.3 \\
\hline & \begin{tabular}{|l|l|l|} 
craft activities \\
\end{tabular} & 408.25 & 16.9 & 15.4 & 1.3 & 0.6 & 34.2 & 67.5 & 43.3 \\
\hline & \begin{tabular}{|l|} 
removable \\
appliance
\end{tabular} & 83.2 & 14.9 & 10.5 & & & 25.5 & 72.1 & 28.4 \\
\hline & \begin{tabular}{|l|} 
fixed appliance \\
\end{tabular} & 325 & 20.3 & 15.8 & 2.3 & 0.4 & 38.7 & 71.5 & 50 \\
\hline & \begin{tabular}{|l|}
$\begin{array}{l}\text { medical } \\
\text { examination }\end{array}$ \\
\end{tabular} & 228.3 & 21.7 & 13.3 & 0.7 & & 35.8 & 75.3 & 41.4 \\
\hline & \begin{tabular}{|l|}
$\begin{array}{l}\text { contra-angle/ } \\
\text { ultrasound }\end{array}$ \\
\end{tabular} & 202.8 & 10.6 & 25 & 12.5 & 0.5 & 48.6 & 69.9 & 56.5 \\
\hline \multirow{6}{*}{$\begin{array}{c}\text { Torso } \\
\text { inclination } \\
\text { (T_i } 1)\end{array}$} & $\begin{array}{l}\begin{array}{l}\text { archwire / elastics } \\
\text { change }\end{array} \\
\end{array}$ & 705.3 & 1.3 & 1.4 & & & 2.7 & 4.7 & 14.4 \\
\hline & \begin{tabular}{|l|l} 
craft activities \\
\end{tabular} & 408.25 & 1.8 & 1.7 & 0.3 & & 3.8 & 7.4 & 11.7 \\
\hline & \begin{tabular}{|l|}
$\begin{array}{l}\text { removable } \\
\text { appliance }\end{array}$ \\
\end{tabular} & 83.2 & 4.2 & 1.3 & & & 5.5 & 13.6 & 12.2 \\
\hline & fixed appliance & 325 & 4.4 & 4.4 & 1.1 & 0.3 & 10.2 & 14.3 & 29.6 \\
\hline & $\begin{array}{l}\text { medical } \\
\text { examination }\end{array}$ & 228.3 & 3.3 & 1.8 & & & 5.1 & 9.7 & 13.9 \\
\hline & $\begin{array}{l}\text { contra-angle/ } \\
\text { ultrasound }\end{array}$ & 202.8 & 2.1 & 6.3 & 4 & 1.1 & 13.5 & 16.1 & 25.4 \\
\hline \multirow{6}{*}{$\begin{array}{l}\text { Torso lateral } \\
\text { inclination } \\
\text { (T_li) }\end{array}$} & $\begin{array}{l}\begin{array}{l}\text { archwire / elastics } \\
\text { change }\end{array} \\
\end{array}$ & 705.3 & 4.1 & 3.7 & 0.6 & 0.1 & 8.6 & 13.4 & 44.8 \\
\hline & \begin{tabular}{|l|l|l|l|} 
craft activities \\
\end{tabular} & 408.25 & 1.7 & 1.9 & 0.1 & & 3.8 & 7.8 & 17.3 \\
\hline & \begin{tabular}{|l|} 
removable \\
appliance
\end{tabular} & 83.2 & 2.1 & 1.5 & & & 3.6 & 8 & 14.4 \\
\hline & \begin{tabular}{|l|} 
fixed appliance \\
\end{tabular} & 325 & 1.5 & 2.2 & 0.2 & & 3.9 & 7.1 & 29.2 \\
\hline & \begin{tabular}{|l|} 
medical \\
examination
\end{tabular} & 228.3 & 4.5 & 3.6 & 0.2 & & 8.4 & 15.5 & 24.8 \\
\hline & $\begin{array}{l}\begin{array}{l}\text { contra-angle/ } \\
\text { ultrasound }\end{array} \\
\end{array}$ & 202.8 & 0.6 & 1.4 & 1.1 & 0.6 & 3.6 & 6.2 & 15.2 \\
\hline \multirow{6}{*}{$\begin{array}{l}\text { Torso torsion } \\
\quad\left(T_{-} t\right)\end{array}$} & $\begin{array}{l}\begin{array}{l}\text { archwire / elastics } \\
\text { change }\end{array} \\
\end{array}$ & 705.3 & 8.4 & 8.2 & 0.8 & 0.2 & 17.6 & 31.7 & 35.3 \\
\hline & \begin{tabular}{|c|} 
craft activities \\
\end{tabular} & 408.25 & 6.6 & 5.9 & 1 & & 13.5 & 26.7 & 25.8 \\
\hline & \begin{tabular}{|l|} 
removable \\
appliance
\end{tabular} & 83.2 & 11.3 & 4.7 & 0.7 & & 16.7 & 36.1 & 24.6 \\
\hline & $\begin{array}{l}\text { fixed appliance } \\
\end{array}$ & 325 & 6.8 & 8.4 & 1.5 & 0.7 & 17.5 & 27.9 & 40.2 \\
\hline & \begin{tabular}{|l|} 
medical \\
examination
\end{tabular} & 228.3 & 9.8 & 10.1 & 1.1 & 0.6 & 21.5 & 36.3 & 31 \\
\hline & $\begin{array}{l}\begin{array}{l}\text { contra-angle/ } \\
\text { ultrasound }\end{array} \\
\end{array}$ & 202.8 & 4 & 11 & 7.2 & 2.7 & 24.9 & 33.8 & 37.4 \\
\hline
\end{tabular}


and $30 \mathrm{~s}, 0 \%-12.5 \%$ are held between 30 and $60 \mathrm{~s}$, and $0 \%-0.6 \%$ are maintained for $60 \mathrm{~s}$ and longer (Table 3, Table 4).

\section{Categories II and III: Office and other activities}

Static postures in category II ("office") were analyzed based on the sub-activity "office work" whereas static postures in category III ("other activities") were analyzed in relation to the sub-activities "conversation" and "taking up/putting down of instruments."

In the head and neck area "office work" (category II) is executed in non-neutral postures with extension/flexion of the cervical spine (CS_f) $57.3 \%$ of the time. This accounts for $32.9 \%$ of the total percentage of static nonneutral postures. The duration of static postures is primarily between 4 and $10 \mathrm{~s}(9.1 \%)$ and $10-30 \mathrm{~s}(8.1 \%)$.
Seventy five percent of postures with extension/ flexion of the Torso (T_f) are in the non-neutral range, from which $54.8 \%$ are executed statically. Based on these results we calculated a total percentage of static postures of $66 \%$. Moreover, we found that $10.1 \%$ of static postures are held for $60 \mathrm{~s}$ or longer, $12.3 \%$ between 30 and $60 \mathrm{~s}, 20.5 \%$ between 10 and $30 \mathrm{~s}$, and $11.9 \%$ have a duration between 4 and $10 \mathrm{~s}$ (Table 5, Table 6).

With regard to category III (other activities) the percentage of non-neutral postures in the head and neck is $<50 \%$ with a conspicuous total percentage of static postures of $<25 \%$ as well as a total percentage of nonneutral postures of $<75 \%$.

The sub-activity "conversation" generated conspicuous results in the extension/flexion of the torso. From

Table 5 Office and other activities: treatment: percentage of neutral, moderate, and awkward postures (\%) of the total duration in the head and neck area as well as the percentage of non-neutral postures as the sum of all moderate and awkward postures (\%). See Table 1 for color-coded ranking system

\begin{tabular}{|c|c|c|c|c|c|c|}
\hline Posture concept $\left[{ }^{\circ}\right]$ & Activity & $\begin{array}{c}\text { Duration } \\
\text { [min] }\end{array}$ & $\begin{array}{r}\text { Percentage of } \\
\text { non-neutral } \\
\text { postures [\% ] }\end{array}$ & $\begin{array}{c}\text { Percentage of } \\
\text { neutral } \\
\text { postures [\%] }\end{array}$ & $\begin{array}{c}\text { Percentage of } \\
\text { moderate } \\
\text { postures }[\%]\end{array}$ & $\begin{array}{r}\text { Percentage of } \\
\text { awkward postures } \\
{[\%]}\end{array}$ \\
\hline \multirow{3}{*}{$\begin{array}{l}\text { Head flexion } \\
\left(\mathrm{H}_{-} \mathrm{f}\right)\end{array}$} & Office work & 1893.7 & 35.6 & 64.4 & 27 & 8.6 \\
\hline & conversation & 1747.4 & 29.6 & 70.4 & 13.8 & 15.8 \\
\hline & $\begin{array}{l}\text { take/ deposit } \\
\text { instrument }\end{array}$ & 158.9 & 49.7 & 50.3 & 42.6 & 7 \\
\hline \multirow{3}{*}{$\begin{array}{l}\text { Head lateral } \\
\text { inclination }\left(\mathrm{H} \_\mathrm{li}\right)\end{array}$} & \begin{tabular}{|l|} 
Office work \\
\end{tabular} & 1893.7 & 26.8 & 73.2 & & 26.8 \\
\hline & \begin{tabular}{|l|} 
conversation \\
\end{tabular} & 1747.4 & 23 & 77 & & 23 \\
\hline & $\begin{array}{l}\text { take/ deposit } \\
\text { instrument } \\
\end{array}$ & 158.9 & 29.6 & 70.4 & & 29.6 \\
\hline \multirow{3}{*}{$\begin{array}{c}\text { Cervical spine } \\
\text { flexion/extension } \\
\text { (CS_f) }\end{array}$} & \begin{tabular}{|l|} 
Office work \\
\end{tabular} & 1893.7 & 57.3 & 42.7 & & 57.3 \\
\hline & conversation & 1747.4 & 50.7 & 49.3 & & 50.7 \\
\hline & $\begin{array}{l}\text { take/ deposit } \\
\text { instrument } \\
\end{array}$ & 158.9 & 42.6 & 57.4 & & 42.6 \\
\hline \multirow{3}{*}{$\begin{array}{l}\text { Cervical spine lateral } \\
\text { flexion (CS_lf) }\end{array}$} & Office work & 1893.7 & 27.6 & 72.4 & & 27.6 \\
\hline & conversation & 1747.4 & 24.1 & 75.9 & & 24.1 \\
\hline & $\begin{array}{l}\text { take/ deposit } \\
\text { instrument }\end{array}$ & 158.9 & 34.2 & 65.8 & & 34.2 \\
\hline \multirow{3}{*}{$\begin{array}{c}\text { Thoracic spine } \\
\text { flexion/extension } \\
\text { (TS_f) }\end{array}$} & \begin{tabular}{|l|} 
Office work \\
\end{tabular} & 1893.7 & 47.3 & 52.7 & 46.4 & 0.9 \\
\hline & conversation & 1747.4 & 24.6 & 75.4 & 24.2 & 0.4 \\
\hline & $\begin{array}{l}\text { take/ deposit } \\
\text { instrument }\end{array}$ & 158.9 & 38.5 & 61.5 & 29.7 & 8.8 \\
\hline \multirow{3}{*}{$\begin{array}{c}\text { Thoracic spine } \\
\text { Lateral flexion } \\
\text { (TS_lf) }\end{array}$} & \begin{tabular}{|l|} 
Office work \\
\end{tabular} & 1893.7 & 3.7 & 96.3 & 3.5 & 0.2 \\
\hline & conversation & 1747.4 & 8.1 & 91.9 & 7.2 & 0.8 \\
\hline & $\begin{array}{l}\text { take/ deposit } \\
\text { instrument } \\
\end{array}$ & 158.9 & 15.1 & 84.9 & 12.8 & 2.3 \\
\hline \multirow{3}{*}{$\begin{array}{c}\text { Torso } \\
\text { flexion/extension } \\
\text { (T_f) }\end{array}$} & \begin{tabular}{|l|} 
Office work \\
\end{tabular} & 1893.7 & 75 & 25 & 39.8 & 35.2 \\
\hline & conversation & 1747.4 & 61.4 & 38.6 & 34 & 27.5 \\
\hline & $\begin{array}{l}\text { take/ deposit } \\
\text { instrument }\end{array}$ & 158.9 & 49 & 51 & 28.2 & 20.8 \\
\hline \multirow{3}{*}{$\begin{array}{l}\text { Torso inclination } \\
\left.\text { ( } \mathrm{T} \_\mathrm{i}\right)\end{array}$} & Office work & 1893.7 & 4.8 & 95.2 & 4.7 & 0.2 \\
\hline & conversation & 1747.4 & 3.2 & 96.8 & 3.1 & 0.1 \\
\hline & $\begin{array}{l}\text { take/ deposit } \\
\text { instrument } \\
\end{array}$ & 158.9 & 22.5 & 77.5 & 20.5 & 2 \\
\hline \multirow{3}{*}{$\begin{array}{c}\text { Torso lateral } \\
\text { inclination (T_li) }\end{array}$} & \begin{tabular}{|l|} 
Office work \\
\end{tabular} & 1893.7 & 2.2 & 97.8 & 2.1 & 0.1 \\
\hline & conversation & 1747.4 & 5.1 & 94.9 & 4.6 & 0.5 \\
\hline & $\begin{array}{l}\text { take/ deposit } \\
\text { instrument }\end{array}$ & 158.9 & 11.9 & 88.1 & 10.4 & 1.4 \\
\hline \multirow{3}{*}{ Torso torsion $\left(T_{-} t\right)$} & \begin{tabular}{|l|} 
Office work \\
\end{tabular} & 1893.7 & 31.1 & 68.9 & 21.9 & 9.2 \\
\hline & conversation & 1747.4 & 28.4 & 71.6 & 19.8 & 8.6 \\
\hline & $\begin{array}{l}\text { take/ deposit } \\
\text { instrument }\end{array}$ & 158.9 & 39.5 & 60.5 & 26.8 & 12.7 \\
\hline
\end{tabular}


Table 6 Percentage of static postures held for $\geq 4$ during office work and other activities. Figure legend: Total percentage of static postures $=$ Sum of all moderate and awkward postures that occur with all activities

\begin{tabular}{|c|c|c|c|c|c|c|c|c|c|}
\hline $\begin{array}{c}\text { Evaluation } \\
\text { parameter }\left[{ }^{\circ}\right]\end{array}$ & Activity & $\begin{array}{c}\text { Durati } \\
\text { on } \\
\text { [min] }\end{array}$ & $\begin{array}{c}\text { Static } \\
\text { postures } \\
4-10 \mathrm{~s} \\
{[\%]}\end{array}$ & $\begin{array}{c}\text { Static } \\
\text { postures1 } \\
0-30 \text { s [\%] }\end{array}$ & $\begin{array}{c}\text { Static } \\
\text { postures } \\
30-60 s \\
{[\%]}\end{array}$ & $\begin{array}{c}\text { Static } \\
\text { posture } \\
s \geq 60 \mathrm{~s} \\
{[\%]}\end{array}$ & $\begin{array}{c}\text { Total } \\
\text { percentag } \\
\text { e of static } \\
\text { postures } \\
{[\%]} \\
\end{array}$ & 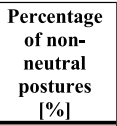 & $\begin{array}{c}\text { Total } \\
\text { percentage of } \\
\text { static non- } \\
\text { neutral } \\
\text { postures [\%] } \\
\end{array}$ \\
\hline \multirow{3}{*}{$\begin{array}{l}\text { Head flexion } \\
\left.\left(\mathrm{H}_{-} \mathrm{f}\right)\right)\end{array}$} & Office work & 1893.7 & 5.8 & 3.9 & 0.8 & 0.4 & 10.9 & 35.6 & 27.1 \\
\hline & conversation & 1747.4 & 2.3 & 0.9 & & & 3.2 & 29.6 & 7.5 \\
\hline & $\begin{array}{l}\text { take/ deposit } \\
\text { instrument }\end{array}$ & 158.9 & 1.3 & 0.5 & & & 1.8 & 49.7 & 2.3 \\
\hline \multirow{3}{*}{$\begin{array}{l}\text { Head lateral } \\
\text { inclination } \\
\text { (H_li) }\end{array}$} & Office work & 1893.7 & 4.7 & 3.2 & 0.7 & 0.2 & 8.9 & 26.8 & 27.3 \\
\hline & conversation & 1747.4 & 2.2 & 1 & 0.2 & & 3.4 & 23 & 10.3 \\
\hline & $\begin{array}{l}\text { take/ deposit } \\
\text { instrument }\end{array}$ & 158.9 & 0.6 & 0.3 & & & 0.9 & 29.6 & 1.2 \\
\hline \multirow{3}{*}{$\begin{array}{l}\text { Cervical spine } \\
\text { flexion/extensi } \\
\text { on }\left(\mathrm{CS}_{-} \mathrm{f}\right)\end{array}$} & Office work & 1893.7 & 9.1 & 8.1 & 2.3 & 0.6 & 20.1 & 57.3 & 32.9 \\
\hline & conversation & 1747.4 & 4 & 1.5 & & & 5.4 & 50.7 & 8.6 \\
\hline & $\begin{array}{l}\text { take/ deposit } \\
\text { instrument }\end{array}$ & 158.9 & 1.1 & 0.5 & & & 1.6 & 42.6 & 2.6 \\
\hline \multirow{3}{*}{$\begin{array}{l}\text { Cervical spine } \\
\text { lateral flexion } \\
\text { (CS_lf) }\end{array}$} & Office work & 1893.7 & 4.5 & 3.4 & 0.7 & 0.3 & 8.9 & 27.6 & 27 \\
\hline & conversation & 1747.4 & 2.3 & 1 & 0.2 & & 3.5 & 24.1 & 9 \\
\hline & $\begin{array}{l}\text { take/ deposit } \\
\text { instrument }\end{array}$ & 158.9 & 0.4 & 0.2 & & & 0.7 & 34.2 & 0.9 \\
\hline \multirow{3}{*}{$\begin{array}{l}\text { Thoracic spine } \\
\text { flexion/extensi } \\
\text { on (TS_f) }\end{array}$} & Office work & 1893.7 & 8 & 13.6 & 7 & 6.5 & 35.1 & 47.3 & 59 \\
\hline & conversation & 1747.4 & 4.9 & 4.7 & 1.3 & 0.3 & 11.2 & 24.6 & 21.1 \\
\hline & $\begin{array}{l}\text { take/ deposit } \\
\text { instrument }\end{array}$ & 158.9 & 3.3 & 1.3 & & & 4.6 & 38.5 & 6.8 \\
\hline \multirow{3}{*}{$\begin{array}{l}\text { Thoracic spine } \\
\text { Lateral flexion } \\
\text { (TS_lf) }\end{array}$} & Office work & 1893.7 & 0.5 & 0.7 & 0.4 & 0.3 & 1.9 & 3.7 & 14.3 \\
\hline & conversation & 1747.4 & 0.8 & 0.9 & 0.4 & 0.3 & 2.4 & 8.1 & 9.9 \\
\hline & $\begin{array}{l}\text { take/ deposit } \\
\text { instrument }\end{array}$ & 158.9 & 1 & 0.6 & 0.4 & & 1.9 & 15.1 & 3.9 \\
\hline \multirow[t]{3}{*}{$\begin{array}{c}\text { Torso } \\
\text { flexion/extensi } \\
\text { on }\left(\mathrm{T} \_ \text {f }\right)\end{array}$} & Office work & 1893.7 & 11.9 & 20.5 & 12.3 & 10.1 & 54.8 & 75 & 66 \\
\hline & conversation & 1747.4 & 11.5 & 10.1 & 2.8 & 1.2 & 25.6 & 61.4 & 30.3 \\
\hline & $\begin{array}{l}\text { take/ deposit } \\
\text { instrument }\end{array}$ & 158.9 & 5.4 & 2.4 & & & 7.7 & 49 & 10.2 \\
\hline \multirow{3}{*}{$\begin{array}{c}\text { Torso } \\
\text { inclination } \\
(\mathrm{T} \text { i })\end{array}$} & Office work & 1893.7 & 1 & 1.6 & 0.4 & 0.1 & 3.1 & 4.8 & 10.4 \\
\hline & conversation & 1747.4 & 0.4 & 0.5 & 0.2 & & 1.1 & 3.2 & 5.7 \\
\hline & $\begin{array}{l}\text { take/ deposit } \\
\text { instrument }\end{array}$ & 158.9 & 2.2 & 0.8 & & & 3.1 & 22.5 & 5.1 \\
\hline \multirow{3}{*}{$\begin{array}{l}\text { Torso lateral } \\
\text { inclination } \\
\text { (T_li) }\end{array}$} & Office work & 1893.7 & 0.3 & 0.5 & 0.3 & 0.1 & 1.1 & 2.2 & 8.3 \\
\hline & conversation & 1747.4 & 0.4 & 0.6 & 0.3 & 0.2 & 1.5 & 5.1 & 8.2 \\
\hline & $\begin{array}{l}\text { take/ deposit } \\
\text { instrument }\end{array}$ & 158.9 & 1 & 0.6 & & & 1.6 & 11.9 & 3.2 \\
\hline \multirow{3}{*}{$\begin{array}{l}\text { Torso torsion } \\
\text { (T_t) }\end{array}$} & Office work & 1893.7 & 4.9 & 8.9 & 4.8 & 3.9 & 22.5 & 31.1 & 47.5 \\
\hline & conversation & 1747.4 & 4.9 & 5.8 & 1.7 & 0.9 & 13.2 & 28.4 & 26.4 \\
\hline & $\begin{array}{l}\text { take/ deposit } \\
\text { instrument }\end{array}$ & 158.9 & 3.8 & 1.2 & & & 5 & 39.5 & 7.4 \\
\hline
\end{tabular}

a total percentage of static postures of $30.3 \%$, participants assumed non-neutral postures $61.4 \%$ of the time. $11.5 \%$ of static postures involved in the subactivity "conversation" had a duration between 4 and $10 \mathrm{~s}, 10.1 \%$ between 10 and $30 \mathrm{~s}, 2.8 \%$ between 30 and $60 \mathrm{~s}$, and $1.2 \%$ had a duration of $60 \mathrm{~s}$ or longer (Table 5, Table 6).

\section{Discussion}

Particular motion patterns are executed repeatedly during daily workflows of orthodontists [34]. These motion patterns can be short-term as well as long-term, dynamic or static. Musculoskeletal disorders in dental professions often originate in static positions that mostly comprise unergonomic sitting postures held incessantly during treatment as well as periodically repeated motions $[22,23,17]$.
With frequently assumed treatment positions the practitioner's body tilts forward whereby the head, neck and torso rotate laterally to gain the best possible view of the inside of patient's mouth. For right-handed subjects, this result in a head rotation to the left and a head flexion to the right [26].

Within this treatment position, data generated in the present study affirms that static postures (4-10 s) in the head and neck area have a shorter duration than static postures in the back, even though we found that in both anatomical areas positions assumed were primarily inclined to the front. Static postures in the back area last between 4 and $10 \mathrm{~s}$ and are almost as frequent as postures that are held between 10 and 30 s. If static postures are assumed for more than $60 \mathrm{~s}$, they primarily refer to restricted and laterally inclined postures of the torso during the sub-activity "contra-angle/ultrasound" (inclination of the thoracic spine to the right (TS_lf), 
inclination of the torso to the right (TS_lf), back torsion to the right (T_r $)$ ). At the same time, we also found a high percentage of static postures of $46.9 \%$ for moderate and unfavorable postures.

As a result, head and neck postures are adjusted in shorter intervals than back postures. The risk of developing work related musculoskeletal disorders is particularly high in the back and neck, a conclusion that is also confirmed by Park et al. [32] and their application of the RULA method.

The back curvature demonstrates similarly high percentage static postures values for activities in category II and III. Nevertheless, the risk that tilted positions bear is smaller with office work as it is an activity executed in supported positions that decrease static muscle strain.

The evaluation of our data on static postures clearly shows that orthodontists remain, especially in the back area, in static anterior inclined postures due to long hours of executing office work and orthodontic treatment. However, ranking static postures, the total duration of the respective activity as well as the percentage of the individual statics components shall be considered. Therefore, we selected only those activities which are the most significant for the orthodontic workflow with regard to duration and frequency (Table 3; Table 5).

The orthodontic treatment of patients is usually an unsupported activity, which results in greater muscular strain. Moreover, it should be noted that particularly results for "office work" can be inaccurate as the measurements conducted could not generate data on support provided by the back of the chair or a wall, for instance. Additional video recording could be used to evaluate the results more profoundly. However, ethical concerns regarding the patient's right to privacy and confidentiality might counter argue the use of video recording as not every orthodontic patient wants to be filmed during treatment.

A Scandinavian study Keruso et al. [44] affirms the most common musculoskeletal disorders among orthodontists. Dentists, orthodontists, and office clerks (control group) were surveyed on health issues and the results of $70 \%$ to $72 \%$ demonstrate that the differences between dentists and orthodontists are rather minor. However, office clerks appear to encounter significantly less musculoskeletal pain as dentists and orthodontists combined.

Despite the similar field of patient treatment, orthodontic activities (check-ups, archwire change, or rebonding brackets) are not identical with general dental activities (restorative fillings, impressions, preparation of dentures, teeth extraction). Although, orthodontists handle more office work than dentists (ORTHO: treatment $34 \%$ vs. office $33 \%$; DDS: $41 \%$ vs. $23 \%$ ), which renders a large share of their treatment theoretical work (model analysis and concise planning of treatment process), they nonetheless execute many activities in anterior inclined and static postures [34]. As a result, orthodontists carry a greater risk of developing work related musculoskeletal disorders due to excessive static stress. Moreover, existing issues with the musculoskeletal system are often related to prolonged static positions [17, 22, 23, 45]. As early as 1972, Schön [25] observed muscular strain in static postures. According to Valachi et al. [26] frequently assumed static positions are more harmful to the human body than dynamic activities. Furthermore, the authors of this study also found that participants assumed static postures more often than dynamic postures. Thereby, more than $50 \%$ of muscles are required to hold a motionless position, which results in fatigue and, with frequent repetition, also in pain. In our opinion, the conclusion of Valachi et al. [28]- is also valid for both, dentists and orthodontists, as their positions during treatment are similar [34].

The kinematic analysis measures the total duration and frequency of static postures conducted by all participants. However, the analysis does not take pauses between the same activities into account. As mentioned earlier, the present data material does not serve to distinguish between supported and unsupported postures. Also, to date no kinematic analysis of fine motor movements in the fingers, hands, and wrists has been conducted yet $(25 \%$ or $44 \%$ suffer from pain in their hands), even though these movements are essential for dental professionals executing concise and delicate tasks $[46,10]$.

\section{Conclusions}

The kinematic analysis of head and torso postures shows a prevalence of static postures in orthodontists. Head and neck postures are adjusted in shorter intervals than back postures. The risk of developing work related musculoskeletal disorders is particularly high in the back, neck and head region. Since most positions of orthodontists during a working day were primarily inclined to the front, i.e. using a "contra-angle" or "ultrasound". Moreover, the study emphasizes that postures ranked as moderate (according to ergonomic norms) paired with static strain can result in restricted postures. These postures present health risks in the workplace as they increase the probability of developing musculoskeletal disorders and are associated with activities on the job. The results of this analysis paired with the ergonomic classification of joint angles into the same categories and activities allow for a quantitative evaluation of the orthodontic profession in relation to the physical strains and the health risks for the musculoskeletal system. 


\section{Abbreviations}

CUELA-System: Computer-assisted acquisition and long-term analysis of musculoskeletal loads; RULA: Rapid upper limb assessment

\section{Acknowledgements}

This article contains parts of the doctoral thesis of Mrs. I. Hauck

\section{Funding}

Not applicable.

\section{Availability of data and materials}

The datasets supporting the conclusions of this article are included within the article.

\section{Authors' contributions}

$\mathrm{DO}, \mathrm{CE}, \mathrm{IH}, \mathrm{JN}$ and DAG made substantial contributions to the conception and design of the manuscript. DO, CE, $\mathrm{H}_{\mathrm{N}} \mathrm{JN}, \mathrm{IH}, \mathrm{RE}, \mathrm{DD}$ and DAG made substantial contributions to the construction of the measurement protocol and DO has been involved in the statistical data analysis. All authors have read and approved the final manuscript.

\section{Competing interests}

The authors declare that they have no competing interests.

\section{Consent for publication}

All individuals have given their consent to publish their images.

\section{Ethics approval and consent to participate}

This study was approved by the Ethics Committee (135/14) of the Goethe University Frankfurt am Main. All participants signed an informed consent to participate in advance.

\section{Publisher's Note}

Springer Nature remains neutral with regard to jurisdictional claims in published maps and institutional affiliations.

\section{Consent to participate}

All participants signed an informed consent to participate in advance.

\section{Author details}

${ }^{1}$ Institute of Occupational Medicine, Social Medicine and Environmental Medicine, Goethe-University, Theodor-Stern-Kai 7, 60590 Frankfurt am Main, Germany. ${ }^{2}$ School of Dentistry, Department of Orthodontics, University Medical Centre of the Johannes Gutenberg University Mainz, Augustusplatz 2, 55131 Mainz, Germany. ${ }^{3}$ Institute for Occupational Health and Safety (IFA) of the German Social Accident Insurance (DGUV), Alte Herrstraße 111, 53757 Sankt Augustin, Germany.

Received: 31 January 2017 Accepted: 15 June 2017

Published online: 23 June 2017

\section{References}

1. Meyer VP, Brehler R, Castro HM, Nentwig CG. Arbeitsbelastungen be Zahnärzten in niedergelassener Praxis Eine arbeitsmedizinische Bestandsaufnahme zu Wirbelsäulenbelastungen, Berufsdermatosen und Stressfaktoren Deutscher Zahnärzte Verlag DAV-Hanser Köln Mündchen Institut der Deutschen Zahnärzte(IDZ): 2001.

2. Gopinadh A, Devi KN, Chiramana S, Manne P, Sampath A, Babu MS. Ergonomics and musculoskeletal disorder: as an occupational hazard in dentistry. J Contemp Dent Pract. 2013;14(2):299-303.

3. Alexopoulos EC, Stathi IC, Charizani F. Prevalence of musculoskeletal disorders in dentists. BMC Musculoskelet Disord. 2004;5:16. doi:10.1186/ 1471-2474-5-16

4. Custodio RA, Silva CE, Brandao JG. Ergonomics work analysis applied to dentistry-a Brazilian case study. Work (Reading Mass). 2012;41(Suppl 1):690-7. doi:10.3233/wor-2012-0227-690.

5. Alghadir A, Zafar H, lqbal ZA. Work-related musculoskeletal disorders among dental professionals in Saudi Arabia. J Physical Ther Sci. 2015;27(4):1107-12. doi:10.1589/jpts.27.1107.

6. Rabiei M, Shakiba M, Shahreza HD, Talebzadeh M. Musculoskeletal disorders in dentists. Int J Occup Hyg. 2012;4:36-40.
7. Rising DW, Bennett BC, Hursh K, Plesh O. Reports of body pain in a dental student population. J Am Dental Assoc (1939). 2005;136(1):81-6.

8. Sankar SG, Reddy PV, Reddy BR, Vanaja KKE. The prevalence of work-related musculoskeletal disorders among Indian orthodontists. J Indian Orthod Soc. 2012:46(4):264-8.

9. Tirgar A, Javanshir K, Talebian A, Amini F, Parhiz A. Musculoskeletal disorders among a group of Iranian general dental practitioners. J Back Musculoskelet Rehabil. 2015;28(4):755-9. doi:10.3233/bmr-140579.

10. Feng B, Liang $Q$, Wang $Y$, Andersen $L L$, Szeto G. Prevalence of work-related musculoskeletal symptoms of the neck and upper extremity among dentists in China. BMJ Open. 2014;4(12):e006451. doi:10.1136/bmjopen2014-006451.

11. Sustova Z, Hodacova L, Kapitan M. The prevalence of musculoskeletal disorders among dentists in the Czech Republic. Acta medica (Hradec Kralove)/Universitas Carolina. Facultas Medica Hradec Kralove. 2013;56(4):150-6.

12. Morse $\mathrm{T}$, Bruneau H, Dussetschleger J. Musculoskeletal disorders of the neck and shoulder in the dental professions. Work (Reading, Mass). 2010:35(4):419-29. doi:10.3233/wor-2010-0979.

13. Dajpratham P, Ploypetch T, Kiattavorncharoen S, Boonsiriseth K. Prevalence and associated factors of musculoskeletal pain among the dental personnel in a dental school. J Med Assoc Thail. 2010;93(6):714-21.

14. Rafeemanesh E, Jafari Z, Kashani FO, Rahimpour F. A study on job postures and musculoskeletal illnesses in dentists. Int J Occup Med Environ Health. 2013;26(4):615-20. doi:10.2478/s13382-013-0133-z.

15. Rohmert W, Mainzer J, Zipp P. Der Zahnarzt im Blickfeld der Ergonomie. Köln: Deut Ärzte Verlag; 1986

16. Blanc D, Farre $P$, Hamel O. Variability of musculoskeletal strain on dentists: an electromyographic and goniometric study. Int J Occup Saf Ergon. 2014·20(2):295-307 doi:10.1080/10803548.2014.11077044

17. Kierklo A, Kobus A, Jaworska M, Botulinski B. Work-related musculoskeletal disorders among dentists - a questionnaire survey. Ann Agric Environ Med. 2011;18(1):79-84.

18. Brown J, Burke FJ, Macdonald EB, Gilmour H, Hill KB, Morris AJ, et al. Dental practitioners and ill health retirement: causes, outcomes and reemployment. Br Dent J. 2010;209(5):E7. doi:10.1038/sj.bdj.2010.813.

19. Burke FJ, Main JR, Freeman R. The practice of dentistry: an assessment of reasons for premature retirement. Br Dent J. 1997;182(7):250-4.

20. Rafie F, Zamani Jam A, Shahravan A, Raoof M, Eskandarizadeh A. Prevalence of upper extremity musculoskeletal disorders in dentists: symptoms and risk factors. J Environ Public Health. 2015;2015:517346. doi:10.1155/2015/517346.

21. Ohlendorf D, Erbe C, Hauck I, Nowak J, Hermanns I, Ditchen D, et al. Kinematic analysis of work-related musculoskeletal loading of trunk among dentists in Germany. BMC Musculoskelet Disord. 2016;17(1):427. doi:10.1186/ s12891-016-1288-0.

22. Hayes M, Cockrell D, Smith DR. A systematic review of musculoskeletal disorders among dental professionals. Int J Dent Hyg. 2009;7(3):159-65. doi:10.1111/j.1601-5037.2009.00395.x

23. Shirzaei M, Mirzaei R, Khaje-Alizade A, Mohammadi M. Evaluation of ergonomic factors and postures that cause muscle pains in dentistry students' bodies. J Clin Exp Dent. 2015;7(3):e414-8. doi:10.4317/jced.51909.

24. Landau K, Pressel G. Medizinischen Lexikon der beruflichen Belastungen und Gefährdungen. Stuttgart: Alfons W. Genter verlag GmbH \& Co. KG; 2009.

25. Schön F. Teamarbeit in der zahnärztlichen Praxis. Berlin: Buch- und Zeitschriften-Verlag die Quintessenz; 1972.

26. Valachi B, Valachi K. Mechanisms leading to musculoskeletal disorders in dentistry. J Am Dent Assoc (1939). 2003:134(10):1344-50.

27. Rundcrantz BL, Johnsson B, Moritz U. Pain and discomfort in the musculoskeletal system among dentists. A prospective study. Swed Den J. 1991;15(5):219-28.

28. Valachi B, Valachi K. Preventing musculoskeletal disorders in clinical dentistry: strategies to address the mechanisms leading to musculoskeletal disorders. J Am Den Assoc (1939). 2003:134(12):1604-12.

29. Delleman NJ, Haslegrave CM, Chaffin DB. Working postures and movements: tools for evaluation and engineering. Boca Raton, London, New York, Washington D.C: CRC Press; 2004

30. Standardisation IOf. ISO 11226 ergonomics-evaluation of static working postures. Geneva: International Organization for Standardisation; 2000.

31. MCAtamney L, Nigel CE. RULA: a survey method for the investigation of work-related upper limb disorders. Appl Ergon. 1993;24(2):91-9. 
32. Park HS, Kim J, Roh HL, Namkoong S. Analysis of the risk factors of musculoskeletal disease among dentists induced by work posture. J Phys Ther Sci. 2015;27(12):3651-4. doi:10.1589/jpts.27.3651.

33. Golchha V, Sharma P, Wadhwa J, Yadav D, Paul R. Ergonomic risk factors and their association with musculoskeletal disorders among Indian dentist: a preliminary study using rapid upper limb assessment. Indian J Dent Res. 2014;25(6):767-71. doi:10.4103/0970-9290.152202.

34. Nowak J, Erbe C, Hauck I, Groneberg DA, Hermanns I, Ellegast R, et al. Motion analysis in the field of dentistry: a kinematic comparison of dentists and orthodontists. BMJ Open. 2016;6(8):e011559. doi:10.1136/bmjopen2016-011559.

35. Ellegast RP. Personengebundenes Messsystem zur automatisierten Erfassung von Wirbelsäulenbelastungen bei beruflichen Tätigkeiten. BIAReport 5/1998. 1998.

36. Ellegast RP. Portable posture and motion measuring system for use in ergomomic field analysis. Stuttgart: Ergon: Ergonomic Software Tools in Product and Workplace Design; 2000. p. 47-54.

37. Freitag S, Fincke-Junod I, Seddouki R, Dulon M, Hermanns I, Kersten JF, et al. Frequent bending-an underestimated burden in nursing professions. Ann Occup Hyg. 2012;56(6):697-707. doi:10.1093/annhyg/mes002.

38. Glitsch U, Ottersbach HJ, Ellegast R, Schaub K, Franz G, Jäger M. Physical workload of flight attendants when pushing and pulling trolleys aboard aircraft. Int J Ind Ergon. 2007;37(11-12):845-54. doi:10.1016/j.ergon.2007.07.004.

39. Kiermayer C, Hoehne-Huckstadt UM, Brielmeier M, Brutting M, Ellegast R, Schmidt J. Musculoskeletal load in and highly repetitive actions of animal facility washroom employees. J Am Assoc Lab Anim Sci. 2011;50(5):665-74.

40. Mache S, Scutaru C, Vitzthum K, Gerber A, Quarcoo D, Welte T, et al. Development and evaluation of a computer-based medical work assessment programme. J Occup Med Toxicol (London, England). 2008;3:35. doi:10.1186/1745-6673-3-35.

41. Mache S, Groneberg DA. Medical work assessment in German hospitals: a real-time observation study (MAGRO) - the study protocol. J Occup Med Toxicol (London, England). 2009;4:12. doi:10.1186/1745-6673-4-12.

42. DIN EN 1005-1. Sicherheit von Maschinen-Menschliche körperliche Leistung-Teil 1. Begriffe Berlin: Beuth Verlag GmbH; 2002.

43. Rosendal L, Langberg $H$, Skov-Jensen A, Kjaer M. Incidence of injury and physical performance adaptations during military training. Clin J Sport Med. 2003;13(3):157-63.

44. Kerosuo E, Kerosuo H, Kanerva L. Self-reported health complaints among general dental practitioners, orthodontists, and office employees. Acta Odontol Scandinavica. 2000;58(5):207-12.

45. Anghel M, Argesanu V, Talpos-Niculescu C, Lungeanu D. Musculoskeletal disorders (MSDS)-consequences of prolonged static postures. J Exp Med Surg Res. 2007. http://jmed.ro/articole/104.pdf. Accessed 31 Jan 2017.

46. Movahhed T, Ajami B, Soltani M, Shakeri MT, Dehghani M. Musculoskeletal pain reports among Mashhad dental students, Iran. Pak J Biological Sci. 2013;16(2):80-5.

\section{Submit your next manuscript to BioMed Central and we will help you at every step:}

- We accept pre-submission inquiries

- Our selector tool helps you to find the most relevant journal

- We provide round the clock customer support

- Convenient online submission

- Thorough peer review

- Inclusion in PubMed and all major indexing services

- Maximum visibility for your research

Submit your manuscript at www.biomedcentral.com/submit 